\title{
Breakdown Driven by Magnetic Field in Gradually Aged Ferrofluid
}

\author{
J. KurimskÝ $\bar{y}^{a, *}$, M. RajñáK ${ }^{a, b}$, R. Cimbala $^{a}$, I. Kolcunováa ${ }^{a}$, B. Dolník ${ }^{a}$, J. DŽmura $^{a}$, \\ J. Petráś $\check{S}^{a}$, J. ZBojovskÝ ${ }^{a}$, P. BARTKO ${ }^{a}$, M. IVAnČÁK ${ }^{a}$, M. TimkO $^{b}$ And P. KopČAnskÝ ${ }^{b}$ \\ ${ }^{a}$ Technical University of Košice, Letná 9, 04154 Košice, Slovakia \\ ${ }^{b}$ Institute of Experimental Physics SAS, Watsonova 47, 04001 Košice, Slovakia
}

\begin{abstract}
This study deals with a specific problem of the impact of magnetic field on the breakdown voltage in gradually aged ferrofluid. The examination was conducted on the samples containing a certain volume of mineral oil with several volume concentrations of iron oxide superparamagnetic nanoscaled particles with surface modified by oleic acid. It is known that certain concentrations of particles in dielectrical insulating oil can improve its electrical withstanding capability. However, the improvement is related to specific operating conditions. In the context of the current research we report a more complex study where the external magnetic field was applied in breakdown voltage tests conducted with the ferrofluid thermally aged for three consecutive $200 \mathrm{~h}$ aging periods. The test fixture was designed for the experimental purpose. During the observation, the DC low-level external magnetic field was applied. The interaction of dispersed particles with external electric and magnetic field resulted in a change of the expected breakdown voltages. We present the breakdown voltage variations depending on two-parameters: the thermal aging and application of external magnetic field. The measurements are analyzed statistically. The ability of the particles to act as space charge accumulators at each stage of the experiment is proven.
\end{abstract}

DOI: 10.12693/APhysPolA.137.939

PACS/topics: magnetic fluid, dielectric breakdown, magnetic field

\section{Introduction}

Ferrofluids (FF) are intensively examined material due to their wide potential of applications. Under external magnetic field a ferrofluid has to stay uniform, with particles not separated from a carrier liquid [1]. Today, FFs are used in electronic parts, detectors of magnetic domain structures, biomedical applications, seals, etc. [2]. A comprehensive physical analysis of FFs can be found in a book by Rosensweig [3]. Interestingly, the FFs have a potential to be applied in electrical power energy industry, particularly, as a possible cooling and insulating fluid in electrical power transformers.

Certain small volume concentration of surface modified iron oxide $\left(\mathrm{Fe}_{3} \mathrm{O}_{4}\right)$ superparamagnetic nanoscaled particles in electrical insulating transformer oil influences not only the thermal transport but its dielectric response $[4,5]$ and breakdown dielectric voltage (BDV) [6-8].

Detailed knowledge of breakdown voltage at various lifecycle stages is an essential parameter for the assessment of the particular application feasibility. In FF, the breakdown streamer development is different than the one in pure carrier oil. The particles play a specific role as they trap the ionization charge and reduce its effective mobility. The result is a slower streamer growth in ferrofluids and higher BDV [9]. The lightening impulse withstand of magnetite $\mathrm{FF}$ was found to be almost twice

\footnotetext{
*corresponding author; e-mail: juraj.kurimsky@tuke.sk
}

of that of the base oil [10]. Related to AC fields, it has been shown that the presence of external magnetic field moderates electrical withstand in FF [11].

A dominant factor changing the insulating fluid quality in power transformer is the heat energy produced in loaded windings. At rated load the average winding temperature rise is less than $65^{\circ} \mathrm{C}$ over the ambient one while top-oil temperature rise cannot exceed $60^{\circ} \mathrm{C}$ [12]. However, the direct access of air to the heated fluid during the accelerated thermal aging test can lead to its complete decomposition [13, 14].

In this paper, we present a comparative study of BDV development in FF during accelerated thermal aging. The external magnetic field is applied to a measuring cell in parallel or perpendicular directions. Supported by statistical analysis we provide the BDV probability estimation to show how the risk of statistical failure develops in aged FF.

\section{Experiment design}

Dielectric properties of examined ferrofluids are derived from base mineral oil with conductivity $10^{-13} \mathrm{~S} / \mathrm{m}$ and dissipation factor $10^{-3}$ at $90^{\circ} \mathrm{C}$. Regarding nanoparticles applied into the colloidal mixture, $\mathrm{Fe}_{3} \mathrm{O}_{4}$ nanoparicles were used, prepared by precipitation method and stabilized by oleic acid $\left(\mathrm{C}_{18} \mathrm{H}_{34} \mathrm{O}_{2}, 65-88 \%\right.$, Merck). Asprepared ferrofluid yields the saturation magnetization $26.6 \mathrm{~A} \mathrm{~m}^{2} / \mathrm{kg}$, as published in our previous work [15].

The average particle core size was found to be $10 \mathrm{~nm}$ (without the surfactant shell), as confirmed by transmission electron microscopy. In the experiment, three volume concentrations of nanoparticles in carrier oil were prepared, $0.05,0.15$, and $0.35 \mathrm{vol} \%$,respectively. 

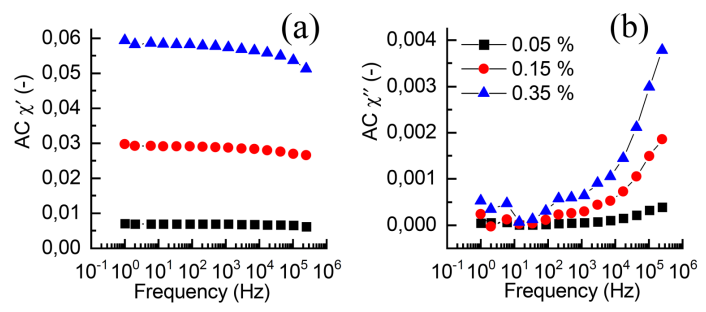

Fig. 1. Real (a) and imaginary (b) part of the AC magnetic susceptibility of the studied ferrofluid samples at room temperature.
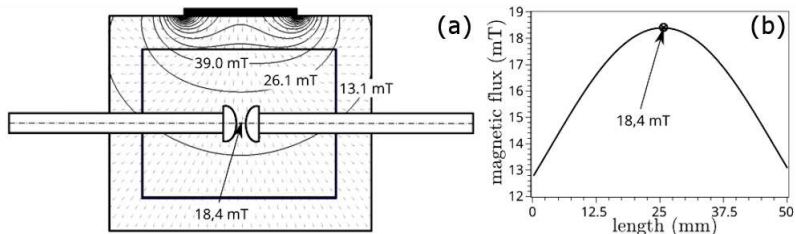

Fig. 2. Experimental test cell arrangement with depicted magnetic flux (black solid lines) and magnetic field distribution (gray petite arrows), top view (a), and magnetic flux density at the electrode radial symmetry axis (b).

AC magnetic susceptibility of the samples is presented in Fig. 1. The absence of complete relaxation maxima in the applied frequency range confirms the small size of the nanoparticles, preferably relaxed by the Néel mechanism. Conditional thermal aging of the samples at $90^{\circ} \mathrm{C}$ was conducted for three time periods: 200, 400, and $600 \mathrm{~h}$, respectively. After each period, BDV tests were performed.
In the experimental design, the measurements followed the recommendations of the international standard IEC 60156. However, due to smaller volume of available ferrofluids, a modified testing cell arrangement was designed. Thus, a volume of approximately $90 \mathrm{ml}$ of FF was enough for testing. Test cell consisted of acrylic glass vessel (inner dimensions in $\mathrm{mm}$ : $40 \times 52.5 \times 52.5$ ) and two semisphere brass electrodes ( $5 \mathrm{~mm}$ in diameter) separated by $0.5 \mathrm{~mm}$ each from other, see Fig. 2a. The resistivity of the electrodes was $0.075 \mu \Omega \mathrm{m}$. The cell was adapted so that a permanent magnet could be attached to it from the bottom-up or to a side wall. In this way, two configurations of electric versus magnetic field could be obtained: the parallel $(\boldsymbol{B} \|)$ or perpendicular $(\boldsymbol{B} \perp)$, respectively. In the mentioned figure, a parallel configuration is depicted. A sintered neodymium magnet $(\mathrm{NdFeB}$, equivalent dimensions in $\mathrm{mm}: 20 \times 10 \times 2)$ was used with residual flux density of $1.3 \mathrm{~T}$ and relative permeability of 1.05 (data sheet values). By means of finite element method, applying the model geometry and material properties, the estimated magnetic flux in the middle point between the electrodes was found to be approximately $18.4 \mathrm{mT}$, as shown in Fig. 2b. The other configuration yielded a magnetic flux of $12.8 \mathrm{mT}$ at the same point. The simulation was done in Agros2D a multiplatform $\mathrm{C}++$ application for solution of partial differential equations (PDE).

Measurements of the breakdown voltages were conducted by the oil dielectric AC test set DTS-60D (High Voltage, USA). The voltage rise-ramp was set to the rate $2000 \mathrm{~V} / \mathrm{s}$. The setup measurement uncertainty is $\pm 2 \%$.
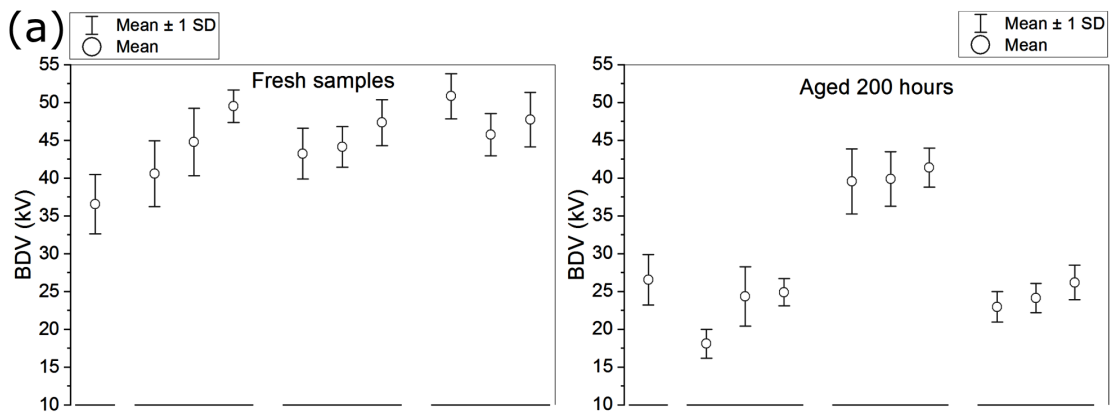

(b)
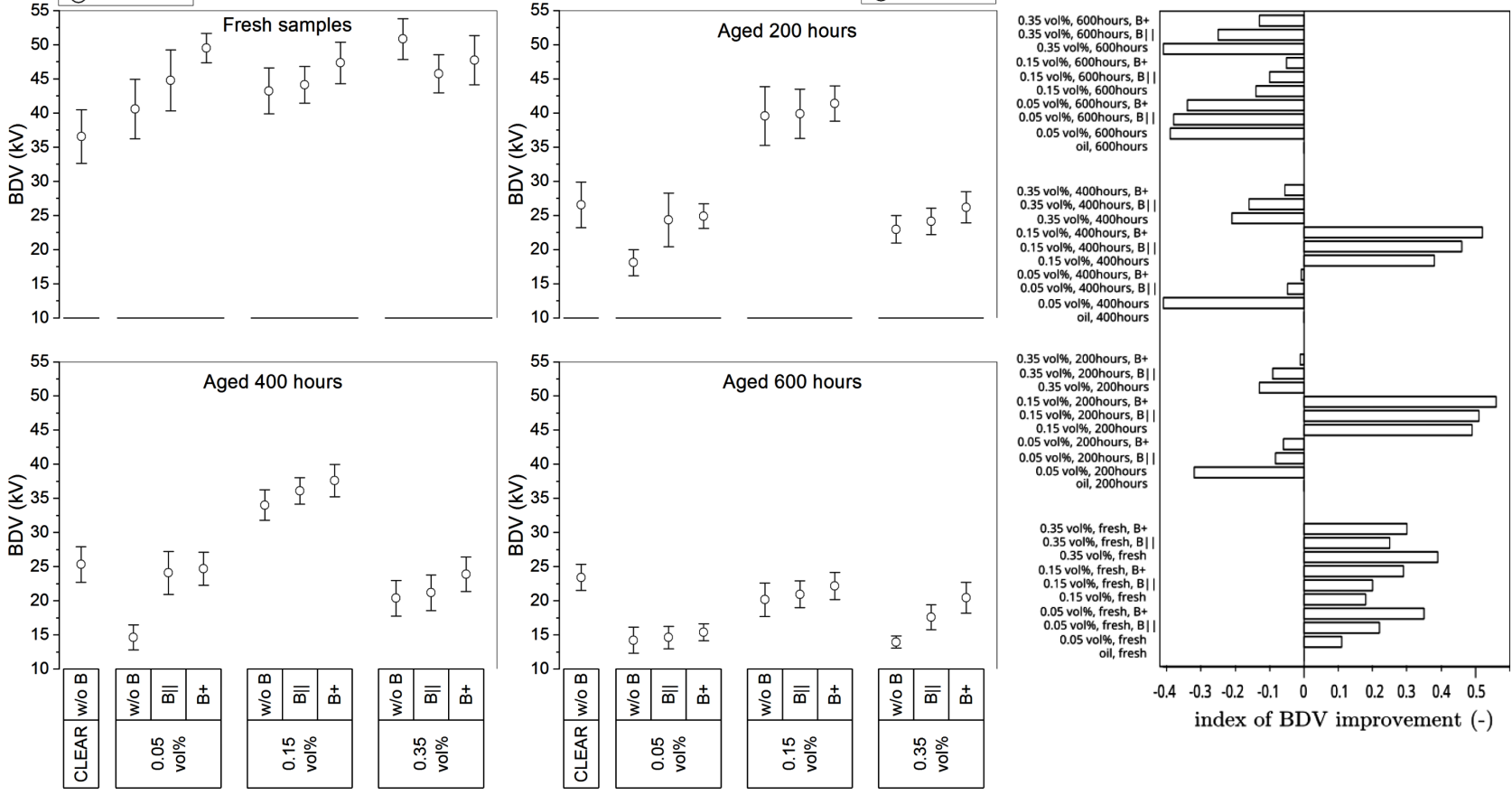

Fig. 3. AC breakdown in ferrofluids of various concentrations in steady magnetic field. 


\section{Results and discussion}

The AC breakdown test was performed for both pure oil and the prepared ferrofluids at each period during the accelerated thermal aging. The results are presented in Fig. 3.

In Fig. 3a, the mean and standard deviation of the measured BDV values are depicted. It is useful to introduce the index of BDV improvement, which can be helpful to clearly characterize the breakdown change related to the base oil. It can be expressed as the ratio of the difference between a particular BDV median of ferrofluid and oil to the one in the clear oil. Graphical interpretation of the index of the BDV improvement is in Fig. 3b.

Generally, the results show that the fresh ferrofluid samples exhibit the improvement in BDV with increasing particle concentration. This implies more effective charge trapping by higher number of the particles. In addition, the external magnetic field enhances the BDV even more. This is associated with the particle-magnetic field interaction, resulting in the hindering effect on the trapped charge migration (streamer formation). Moreover, in the perpendicular configuration, the Lorentz force intensifies the hindering effect. However, as can be seen from Fig. 3, the thermal aging results in remarkable deterioration of the BDV. The effect is ascribed to a gradual destabilization of the particles and subsequent aggregation. The particle aggregates exhibit smaller surface to volume ratio than the individual particles, and therefore less free charge can be trapped in the aggregates. On the other hand, the aggregates of destabilized particles may form conductive paths between the electrodes. When comparing the aging effect on the studied samples, one finds that the ferrofluid of $0.15 \mathrm{vol} . \%$ particle concentration exhibits itself as the best among the samples included in the experiment. As each sample underwent the same thermal aging conditions, the obtained result is not clear yet and opens further avenues of future research.

\section{Conclusions}

In this work, the breakdown in suspensions of transformer oil with different volume concentrations of magnetite nanoparticles was measured. Expectedly, the results exhibit an improvement in the breakdown strength after the addition of particles into the mineral oil. The improvement is enhanced more after external magnetic field application either in parallel or perpendicular direction with regard to the electric field. The experiment showed the fluid disintegration due to the external thermal field applied for certain time periods. Finally, it resulted in the worsening of the breakdown conditions in the aged ferrofluids, when compared to the base oil. Thus, the presence of magnetic particles and the applied magnetic field enhances the breakdown strength of the oil, but for future applications the ferrofluid preparation procedure should be improved to ensure the colloidal stability against the thermal aging.

\section{Acknowledgments}

Slovak Academy of Sciences and Ministry of Education, projects VEGA No. 1/0340/18 and 2/0011/20. Ministry of Education Agency for structural funds of EU projects ITMS 26220120055, ITMS 313011T565 and COST CA15119 NANOUPTAKE. Slovak Research and Development Agency projects APVV-15-0438 and APVV-18-0160.

\section{References}

[1] S. Odenbach, MRS Bull. 38, 921 (2013).

[2] A. Pietrikova, M. Kravcik, in: Proc. 2012 35th Int. Spring Seminar on Electronics Technology, Bad Aussee (Austria), 2012, p. 138.

[3] R.E. Rosensweig, Ferrohydrodynamics, Courier Corporation, 1997.

[4] Š. Hardoň, J. Kúdelčík, P. Bury, M. Gutten, Acta Phys. Pol. A 133, 477 (2018).

[5] Š. Hardoň, J. Kúdelčík, E. Jahoda, M. Kúdelčíková, Int. J. Thermophys. 40, 24 (2019).

[6] V. Segal, A. Rabinovich, D. Nattrass, K. Raj, A. Nunes, J. Magn. Magn. Mater. 215-216, 513 (2000).

[7] J. Fal, O. Mahian, G. Żyła, Energies 11, 2942 (2018).

[8] Y. Lv, M. Rafiq, C. Li, B. Shan, Energies 10, 1025 (2017).

[9] J.G. Hwang, M. Zahn, F.M. OSullivan, L.A.A. Pettersson, O. Hjortstam, R. Liu, J. Appl. Phys. 107, 014310 (2010).

[10] V. Segal, A. Hjortsberg, A. Rabinovich, D. Nattrass, K. Raj, in: Conf. Rec. 1998 IEEE Int. Symp. on Electrical Insulation, Arlington (VA), 1998, Vol. 2, p. 619 .

[11] M. Rajnak, J. Kurimsky, B. Dolnik, K. Marton, L. Tomco, M. Molcan, P. Kopcansky, M. Timko, Acta Phys. Pol. A 126, 248 (2014).

[12] W.H. Tang, Q.H. Wu, Condition Monitoring and Assessment of Power Transformers Using Computational Intelligence, Springer Sci. \& Business Media, 2011.

[13] V. Segal, D. Nattrass, K. Raj, D. Leonard, J. Magn. Magn. Mater. 201, 70 (1999).

[14] J. Kurimský, M. Rajňák, P. Bartko, K. Paulovičová, R. Cimbala, D. Medved', M. Džamová, M. Timko, P. Kopčanský, J. Magn. Magn. Mater. 465, 136 (2018).

[15] M. Rajnak, J. Kurimsky, B. Dolnik, J. Appl. Phys. 114, 034313 (2013). 